\title{
Effect of void cluster on ductile failure evolution
}

Tvergaard, Viggo

Published in:

Meccanica

Link to article, DOI:

10.1007/s11012-016-0537-5

Publication date:

2016

Document Version

Peer reviewed version

Link back to DTU Orbit

Citation (APA):

Tvergaard, V. (2016). Effect of void cluster on ductile failure evolution. Meccanica, 51(12), 3097-3105. https://doi.org/10.1007/s11012-016-0537-5

\section{General rights}

Copyright and moral rights for the publications made accessible in the public portal are retained by the authors and/or other copyright owners and it is a condition of accessing publications that users recognise and abide by the legal requirements associated with these rights.

- Users may download and print one copy of any publication from the public portal for the purpose of private study or research.

- You may not further distribute the material or use it for any profit-making activity or commercial gain

- You may freely distribute the URL identifying the publication in the public portal

If you believe that this document breaches copyright please contact us providing details, and we will remove access to the work immediately and investigate your claim. 


\title{
EFFECT OF VOID CLUSTER ON DUCTILE FAILURE EVOLUTION
}

\author{
Viggo Tvergaard \\ Department of Mechanical Engineering, Solid Mechanics \\ Technical University of Denmark, DK-2800 Kgs. Lyngby, Denmark
}

\begin{abstract}
The behavior of a non-uniform void distribution in a ductile material is investigated by using a cell model analysis to study a material with a periodic pattern of void clusters. The special clusters considered consist of a number of uniformly spaced voids located along a plane perpendicular to the maximum principal tensile stress. A plane strain approximation is used, where the voids are parallel cylindrical holes. Clusters with different numbers of voids are compared with the growth of a single void, such that the total initial volume of the voids, and thus also the void volume fractions, are the same for the clusters and the single void. In the comparison it is essential that local void coalescence inside the clusters is accounted for, since this allows for considering the rate of growth of the single larger void that results from coalescence in the cluster. To obtain a parametric understanding, different transverse stresses on the unit cell are considered to see the influence of different levels of stress triaxiality. Also considered are different initial ratios of the void spacing to the void radius inside the clusters. And results are shown for different levels of strain hardening in the material.
\end{abstract}

KEYWORDS: Void cluster, coalescence, large strain plasticity, ductile fracture.

\section{INTRODUCTION}

When a ductile material deforms under relatively high hydrostatic tension micro-voids contained in the material tend to grow large and ductile failure will occur by coalescence of neighbouring voids. Early models for void growth in such situations were developed by McClintock (1968) and Rice and Tracey (1969), and subsequently a large amount of research has focussed on this area (see reviews by Garrison and Moody, 1987; Tvergaard, 1990; Benzerga and Leblond, 2010; Benzerga et al., 2016). The most widely known porous ductile material model is that developed by Gurson (1977) and subsequently extended (Tvergaard, 1981; Tvergaard and Needleman, 1984). This model is only a good approximation when the stress triaxiality is rather high, as it assumes that the voids remain spherical.

At lower stress triaxiality voids will tend to elongate in the tensile direction. The effect of the initial void shape and of void shape evolution has been studied by a number of authors (e.g. Gologanu et al., 1997; Pardoen et al., 2000; Danas et al., 2009a,b), including also effects of the initial void shape in shear fields (Scheyvaerts et al., 2006; 2011). Under sufficiently low hydrostatic tension, such as in simple shear, the voids close up completely and form micro-cracks, as has been studied in a number of plane strain cell model analyses 
for a material containing a periodic array of circular cylindrical voids Tvergaard (2008, 2009, 2012) or spherical voids (Nielsen et al., 2012). Subsequently the micro-cracks rotate and elongate until interaction with neighbouring micro-cracks gives coalescence. Thus, also in simple shear ductile failure occurs due to the deformation of voids and their interaction with neighbouring voids, but the mechanism is very different from the well-known void growth to coalescence mechanism under tensile loading.

A number of studies have considered the effect of non-uniform distances between voids. Geltmacher et al. (1996) have considered thin metal sheets under either uniaxial or equalbiaxial tension, with various random distributions of through-thickness holes. For the material in front of a crack-tip random void distributions, represented as islands of void nucleating particles in a Gurson material, have been analysed by Tvergaard and Needleman (1992, 2006) both in plane strain and in full 3D. It is found that first hole linking is promoted by a decreased interhole spacing as occurs in clusters of holes. Thomson et al. $(1999,2003)$ have studied periodic clusters of particles in the form of two closely spaced spherical particles, placed either on a line perpendicular to the maximum principal stress direction, or on an inclined line. The two voids on a line perpendicular to the maximum principal stress direction were found to be most critical. In these studies void nucleation is modelled by a cohesive zone at the interface, or by failure in a secondary population of small voids represented by the Gurson model. Bandstra and Koss (2008) have analysed a cluster of three closely spaced spherical voids, by considering a 3D finite element solution for a triangular material region that contains only part of one void. They have estimated the development towards void coalescence by considering the relatively fast growth of the straining in the ligament between voids, for different levels of stress triaxiality and for different ratios of the void spacing to void diameter.

Experimental studies of void growth to coalescence involve effects of the non-uniform void distributions that occur in practice, as e.g. in the studies of Chiantoni et al. (2014) for a ferritic-martensitic steel ASTM A 335 grade P91 and of Tinet et al. (2004) for a resulferised stainless steel (AISI 303). It is shown that small micro-cracks can develop by local coalescence well before the final catastrophic void coalescence.

The present study of cluster effects considers the interaction of a number of voids located on a line perpendicular to the maximum principal stress direction, as was found by Thomson et al. (1999) to be most critical. The study is carried out in a plane strain context so that the voids are actually parallel circular cylindrical voids. The purpose here is to compare the effect 
of clusters with different numbers of voids, but so that the total initial void volume fraction is the same. Am important aspect of the present analyses is that void coalescence inside the cluster is accounted for, so that the cluster of small voids finally develops into a single larger void. Void growth and coalescence is modelled in a way rather similar to that used in studies of ductile crack growth through discretely modelled voids (Tvergaard and Hutchinson, 2002; Tvergaard, 2007).

\section{PROBLEM FORMULATION AND NUMERICAL PROCEDURE}

The cluster of voids to be analysed is located at the center of a rectangular region with the initial width $2 A_{0}$ in the $x^{1}$-direction and the initial height $2 B_{0}$ in the $x^{2}$-direction. The number of voids in the cluster is denoted $N_{v}$ and the initial void radius is $R_{0}$. Due to symmetries only one quarter of the region is analysed, as shown with the dimensions $A_{0} \times B_{0}$ in Fig. 1b. The cluster of voids is taken to be located near the origin, at $x^{1}=0$, with $N_{v}$ voids aligned on the $x^{1}$-axis, as illustrated in Fig. 1a. The case of Fig. 1a corresponds to $N_{v}=5$, where only half of the voids in the cluster are visible due to the assumed symmetry.

Finite strains are accounted for in the analyses, and a convected coordinate Lagrangian formulation of the field equations is used, with the Cartesian $x^{i}$ coordinate system used as reference and with the displacement components on reference base vectors denoted by $u^{i}$. The metric tensors in the reference configuration and the current configuration, respectively, are $g_{i j}$ and $G_{i j}$ with determinants $g$ and $G$, and $\eta_{i j}=1 / 2\left(G_{i j}-g_{i j}\right)$ is the Lagrangian strain tensor. In terms of the displacement components the Lagrangian strain tensor is

$$
\eta_{i j}=\frac{1}{2}\left(u_{i, j}+u_{j, i}+u_{, i}^{k} u_{k, j}\right)
$$

where ()$_{, j}$ denotes covariant differentiation in the reference frame. The contravariant components $\tau^{i j}$ of the Kirchhoff stress tensor on the current base vectors are related to the components of the Cauchy stress tensor $\sigma^{i j}$ by $\tau^{i j}=\sqrt{G / g} \sigma^{i j}$. For the finite strain formulation for a $J_{2}$ flow theory applied, with the Mises yield surface, the incremental stressstrain relationship takes the form $\dot{\tau}^{i j}=L^{i j k \ell} \dot{\eta}_{k \ell}$, with the instantaneous moduli specified in (Hutchinson, 1973; Tvergaard, 1976). The true stress-logarithmic strain curve in uniaxial tension is taken to follow the power law 


$$
\varepsilon= \begin{cases}\sigma / E, & \sigma \leq \sigma_{Y} \\ \left(\sigma_{Y} / E\right)\left(\sigma / \sigma_{Y}\right)^{n}, & \sigma \geq \sigma_{Y}\end{cases}
$$

with Young's modulus $E$, the initial yield stress $\sigma_{Y}$ and the power hardening exponent $n$. Poisson's ratio is $v$.

In a unit cell analysis where $N_{v}$ is the number of voids in the cluster the initial void volume fraction is

$$
f_{0}=N_{v} \pi R_{0}^{2} /\left(4 A_{0} B_{0}\right)
$$

The purpose here is to compare the void volume growth for different clusters, which all have the same value of the initial void volume fraction $f_{0}$, but different values of $N_{v}$. Thus, for a fixed value of $f_{0}$ the initial void radius $R_{0}$ is different for each cluster.

On the four sides of the quarter unit cell (Fig. 1b) symmetry boundary conditions are applied, so that the sides are kept straight. The two sides located on the coordinate axes are prescribed to remain on the axes, with the incremental boundary conditions

$$
\begin{array}{lllll}
\Delta u_{1}=0 & \text { and } & \Delta T^{2}=0 & \text { at } & x^{1}=0 \\
\Delta u_{2}=0 & \text { and } & \Delta T^{1}=0 & \text { at } & x^{2}=0
\end{array}
$$

where $\Delta T^{i}$ are the nominal traction increments. The other two sides have prescribed displacements $U_{1}$ or $U_{2}$ in the directions normal to the sides, with the incremental boundary conditions

$$
\begin{array}{lllll}
\Delta u_{1}=\Delta U_{1} & \text { and } & \Delta T^{2}=0 & \text { at } & x^{1}=A_{0} \\
\Delta u_{2}=\Delta U_{2} & \text { and } & \Delta T^{1}=0 & \text { at } & x^{2}=B_{0}
\end{array}
$$

On the void surfaces the tractions are zero. Then, the average logarithmic strains in the two coordinate directions are $\varepsilon_{1}=\ln \left(1+U_{1} / A_{0}\right)$ and $\varepsilon_{2}=\ln \left(1+U_{2} / B_{0}\right)$. The average true stresses $\Sigma_{11}$ and $\Sigma_{22}$ in the two coordinate directions are calculated as the averages of the stresses on the relevant edge of the deformed unit cell. In each increment of the solution the displacement increment $\Delta U_{2}$ is prescribed, while the increment $\Delta U_{1}$ is calculated such that the ratio of the macroscopic stresses remains fixed

$$
\Sigma_{11} / \Sigma_{22}=\kappa
$$


By prescribing different values of the constant $\kappa$ the void growth behaviour can be compared at different levels of stress triaxiality.

In the void cluster failure occurs by coalescence of neighbouring voids. The coalescence process is here approximated by releasing the forces in the ligament when it has become quite thin, i.e. when the ratio of the current ligament thickness to the initial ligament thickness has reached the value $\chi_{c}$. When a ligament between two voids has reached this critical thickness, the nodal forces on the ligament are stepped down to zero during a number of subsequent increments. Here, the value of $\chi_{c}$ is taken to be 0.3 . It is noted that the value of $\chi_{c}$ used in an earlier analysis of crack growth through a row of discrete voids (Tvergaard and Hutchinson, 2002) was larger, 0.6, while a smaller value 0.15 was used in a subsequent crack growth study (Tvergaard, 2007). In very pure metals necking sometimes continues down to a point so that a very small value of $\chi_{c}$ would be appropriate, but in many structural alloys the neck typically breaks before that. Here the value $\chi_{c}=0.3$ is chosen as a reasonable approximation.

The numerical solutions for the fields inside the unit cell are obtained by a linear incremental solution procedure, based on the principle of virtual work. By expanding this about the current state, the incremental equation is found as

$$
\int_{V}\left\{\Delta \tau^{i j} \delta \eta_{i j}+\tau^{i j} \Delta u_{, i}^{k} \delta u_{k, j}\right\} d V=\int_{A} \Delta T^{i} \delta u_{i} d A-\left[\int_{V} \tau^{i j} \delta \eta_{i j} d V-\int_{A} T^{i} \delta u_{i} d A\right]
$$

Here, $V$ and $A$ are the volume and surface of the body in the reference configuration, $\Delta \tau^{i j}$ and $\Delta \eta_{i j}$ are the stress and strain increments, $T^{i}$ are contravariant components of the nominal surface tractions, etc. On the void surface the nominal tractions are zero. The bracketed terms are equilibrium corrections. The displacement fields are approximated in terms of 8-noded isoparametric elements, with the volume integral in the incremental principle of virtual work carried out by using $2 \times 2$ point Gauss integration within each element. During the numerical analyses a special Rayleigh-Ritz finite element method (Tvergaard, 1976) is used to maintain the prescribed stress ratio (4) and to be able to prescribe internal displacement increments in the material above voids, so that the solution can be continued through unstable equilibria during the approximate procedure used for the coalescence process. 
Remeshing is used here to avoid severe mesh distortion. The remeshing procedure applied was first introduced in one of the authors finite strain programmes by Pedersen (1998), and has been further developed in (Tvergaard, 1997, 2004). The values of field quantities in the integration points of the new mesh are determined by interpolation in the old mesh. To do this, it is necessary to determine the location of each new nodal point and integration point in the old mesh, i.e. the element number and the appropriate values of the local coordinates $\xi$ and $\eta$ inside that element, where the region of the element is specified by $-1 \leq \xi \leq 1$ and $-1 \leq \eta \leq 1$. This is done by repeated use of a Newton-Raphson iteration. A remeshing is carried out when $\Delta \varepsilon_{e} \geq\left(\Delta \varepsilon_{e}\right)_{\max }$ in any integration point, where $\varepsilon_{e}=\int\left(2 \dot{\eta}_{i j} \dot{\eta}^{i j} / 3\right)^{1 / 2} d t$ is an effective strain measure, and $\Delta \varepsilon_{e}$ denotes the amount of this strain accumulated since last remeshing. Here, the limiting value $\left(\Delta \varepsilon_{e}\right)_{\max }$ is mostly chosen as 0.4 .

\section{RESULTS}

In the analyses to be carried out here the material parameters are taken to be $\sigma_{Y} / E=0.003$, $v=1 / 3$ and $n=10$. The cell model is chosen to be square, so that $A_{0}=B_{0}$. Furthermore, it is chosen to consider a very small initial void volume fraction $f_{0}=0.1295 * 10^{-5}$. This means that for $N_{v}=1$ the initial void radius is $R_{0}=0.001286 A_{0}$, while $R_{0}=0.000575 A_{0}$ for $N_{v}=5$ and $R_{0}=0.0004286 A_{0}$ for $N_{v}=9$. In the reference calculations to be presented first the initial spacings between voids are $X_{0}=0.02143 A_{0}$.

In the reference calculations (Fig. 3) the ratio of the transverse stress to the main tensile stress is taken to be given by $\kappa=0.25$ in Eq. (4). For three different values of $N_{v}$ Fig. 3a shows the variation of the normalised tensile stress $\Sigma_{22} / \sigma_{Y}$ vs. the tensile logarithmic strain $\varepsilon_{2}$ and Fig. 3b shows the corresponding evolution of the total void volume $V$ normalised by the initial void volume $V_{0}$. In the case of the single void, the stress passes through a flat maximum while the void volume grows by a large factor, reaching $V / V_{0}=1391$ at the end, but even so, due to the very small initial void volume fraction, the volume fraction at the end of the curve is still small, $f=0.0018$. It is noted that the volume change of the unit cell is 
small, resulting only from elastic dilatation and the growth of a very small void volume fraction. Both for $N_{v}=5$ and for $N_{v}=9$ the stress drops significantly during the void coalescence, i.e. while the ligament failure is being modelled by stepping down the nodal forces on the ligament. Subsequently the stress does not quite reach the previous level during the continued growth of the single void that has resulted from coalescence in the original void cluster. In the figure it looks like there is only one load drop, but in fact the ligaments break separately at closely spaced strain values. First the outer ligament breaks then the next one, and last the ligaments closest to the centre of the cluster. It is noted that during the significant stress drops the solution goes through unstable equilibria, which is controlled by the Rayleigh-Ritz finite element method applied. In an experiment under displacement control there would instead be some local dynamics, as the solution would snap.

It is seen in Fig. 3a that the ligaments break earlier in the cluster with $N_{v}=5$ than in the cluster with more voids. However, when the ligaments break in the cluster with $N_{v}=9$ the total void volume grows more steeply. Before the ligaments break in any of the clusters the void volume has grown more rapidly in the case of the single larger void, but after void coalescence the single void grows more slowly than the total void volumes in both clusters. Thus, the conclusion based of Fig. 3 is that for a given initial void volume fraction the final void resulting from a cluster of many voids is going to grow more rapidly, and the corresponding overall stress on the unit cell is going to decay more rapidly.

It is noted that the computations here are not continued to consider final failure of the materials represented by the unit cells, which would involve interaction between voids in neighbouring unit cells. Even though the void volumes grow by a huge factor, as illustrated in Fig. $3 b$, the void volume fractions at the end of the computations are still low, not exceeding $f=0.002$.

Deformed meshes for the computation with $\kappa=0.25$ and $N_{v}=5$ are shown in Figs. 4 and 5. The initial mesh for this computation is that shown in Fig. 2. At the rather early stage, for $\varepsilon_{2}=0.174$ and $V / V_{0}=26.8$, Fig. 4 shows that the ligaments between voids have not yet thinned down enough to reach the critical condition for coalescence ( $\chi_{c}=0.3$ ), but this initiates at a slightly larger strain, where the corresponding curve in Fig. 3b starts to grow steeply. Fig. 5, for $\varepsilon_{2}=0.227$ and $V / V_{0}=1426$, is near the end of the corresponding curves in Fig. 3. The material around the broken ligaments has not deformed much since the occurrence of coalescence, but it can be seen that the ligament closest to the centre has not 
opened quite as much as the outer ligament. This results from the fact that the outer ligament breaks first, as mentioned above in relation to Fig. 3.

The effect of the stress triaxiality level is illustrated in Fig. 6 by comparing results for three different values of the stress ratio $\kappa$. The curves for the reference case $(\kappa=0.25)$, which are also shown in Fig. 3, are included in Fig. 6 for comparison. In addition, curves are shown for uniaxial plane strain tension $(\kappa=0)$, to represent a case of relatively low stress triaxiality, and for a case of much higher stress triaxiality $(\kappa=0.5)$. As expected, the high stress triaxiality gives much more rapid void growth, so here the single void grows large at an early stage, and also in the void clusters coalescence occurs early. By contrast, under uniaxial plane strain tension the voids grow rather slowly, and the large increase of the void volume as well as the coalescence take place at much larger strains. But apart from these differences in failure strain and the corresponding differences in the stress levels during the final rapid void growth the pattern of behaviour is much the same at the different values of $\kappa$. The single void grows more rapidly at first, but after void coalescence the single void resulting from the clusters grows much more steeply, so that the clusters of the same initial void volume fraction will end up having a more detrimental effect.

The effect of the initial void spacing in the clusters is studied in Fig. 7. In the reference calculations the initial void spacing is $X_{0}$, as shown in Fig. 1a. All calculations in Fig. 7 are for $\kappa=0.25$, and the curves for the reference calculation (Fig. 3) are repeated in Fig. 7. The new results here are the two curves for double void spacing, marked $2 X_{0}$, and the two curves for half void spacing, marked $0.5 X_{0}$. All the curves for void clusters refer to the same curve for $N_{v}=1$, since the total void volume is the same in all cases. It is seen that the void spacing does significantly affect the predicted behaviour. As expected, a larger void spacing relative to the initial void radius gives later failure of the ligaments between neighbouring voids, since more growth of each individual void is needed before the voids get close enough to initiate necking of the ligaments. On the other hand, a smaller void spacing leads to earlier onset of ligament failure and thus earlier development of the rapid growth of the single void resulting from coalescence of all voids in the cluster.

The effect of the strain hardening exponent on the cluster behaviour is illustrated in Fig. 8 for $\kappa=0.25$. As expected, much higher stress levels are reached for the more high hardening material with $n=5$. Also, the rapid failure evolution occurs at larger strains for the high hardening material. The opposite trends are shown for the more low hardening 
material, $n=20$, that here the stress levels reached are lower and rapid failure develops at lower strains. However, at each hardening exponent the effect of the clusters follows the pattern also found in Figs. 3 and 6, that the ligaments break first in the cluster with $N_{v}=5$, where the voids are initially larger relative to the void spacing. Also, when the ligaments break in the cluster with $N_{v}=9$ the total void volume grows more steeply than found for the cluster with $N_{v}=5$. For each of the hardening levels the void volume grows initially more rapidly in the case of the single larger void, but after void coalescence the single void grows more slowly than the total void volumes in both clusters.

\section{DISCUSSION}

A number of investigations of the effect of void clustering have considered either solids with random distributions of voids, or solids with chosen patterns of closely spaced voids. The present study, based on a plane strain model, considers a special type of cluster, where a number of voids are placed along a line perpendicular to the main tensile stress. Previous studies have shown that this orientation of closely spaced voids is more critical than orientations inclined to the main principal stress direction. It is chosen to consider clusters with either 5 or 9 equally spaced voids, and to compare the rate of growth with that of a single void, where both the clusters and the single void represent the same initial total void volume fraction.

A full 3D model with spherical or ellipsoidal voids would be more realistic than the present plane strain analyses. But with the corresponding number of voids in the two in-plane directions this would require a large mesh, so that the analyses would be computationally heavy. However, it is noted that the cluster analyses by Bandstra and Koss (2008) are full 3D, considering a small number of voids, and the 3D analyses of Tvergaard and Needleman (2006) account for many discrete voids represented as islands of void nucleating particles in a Gurson material.

The stress states considered range from uniaxial plane strain tension with rather low stress triaxiality to high transverse tension, resulting in more rapid void growth under the much higher stress triaxiality. Also the spacing between the voids has been varied, and it is found that for the same initial void size a smaller spacing gives earlier void coalescence in the clusters and thus earlier onset of the rapid growth of the single larger void resulting from 
coalescence of all the voids in the cluster. When comparing a low hardening material against a high hardening material, it is found that higher stress levels are reached for high hardening where also the rapid void growth develops later. In all the cases analysed the volume of the single void grows initially more rapidly than the total volume of the voids in a cluster, but after void coalescence the total void volumes in both clusters grow more rapidly, and the indication is that the clusters will lead to earlier material failure, even though the initial void volumes were the same in all the cases analysed here. Local void coalescence occurs earlier in the clusters with 5 voids than in those with 9 voids, because the 9 voids are smaller for the same total volume, and the spacings are the same.

It is noted that the initial void volume fractions are so small that even at the end of the computations, where the void volume has grown by a factor around 1500, the void volume fraction is only around 0.002 , so the studies have not approached final ductile fracture. The present unit cell model studies represent a material with periodically distributed void clusters. The cell models have the initial dimensions $2 A_{0}$ in both coordinate directions, and due to symmetries only one quarter of the cell is analysed. In a final ductile failure situation for this material the voids at neighbouring clusters would come so close that they would interact in ductile failure mechanisms, involving either necking of the ligaments between the voids or shear band localization with void-sheet fracture. But the initial void spacings in the clusters considered here are so small compared to the cell dimensions that there will only be little interaction between neighbour clusters at the end of the present computations. Thus, the comparisons of different clusters here are entirely focussed on the initial stage of void growth, in order to see how the behaviour of clusters compares with that of single voids, when the total initial void volume fraction is the same. Essential in this comparison is that void coalescence inside the clusters is accounted for, so that the stage is reached where the cluster grows as a single larger void.

A disk shaped cluster normal to the maximum tensile stress direction has also been studied by Ohno and Hutchinson (1984) in an axisymmetric solution, with the cluster represented by a Gurson material. Here the focus was on determining when the effect of the cluster is strong enough to give plastic flow localization in the band containing the cluster.

Funding: This study was partially supported by the Technical University of Denmark. Conflict of interest: The author declares that he has no conflict of interest. 


\section{REFERENCES}

Bandstra, J.P., Koss, D.A. (2008) On the influence of void clusters on void growth and coalescence during ductile fracture. Acta Materialia, 56:4429-4439.

Benzerga, A.A., Leblond, J.-B. (2010) Ductile fracture by void growth to coalescence. Advances in Applied Mechanics, 44:169-305.

Benzerga, A.A., Leblond, J.-B., Needleman, A., Tvergaard, V. (2016) Ductile failure modelling. Int. J. Fracture (in press).

Chiantoni, G., Comi, C., Mariani, S., Bonora, N. (2014) Experimental assessment of ductile damage in P91 steel at high temperature. Int. J. Damage Mechanics 23(4):567-587.

Danas, K., Castaneda, P. Ponte. (2009a) A finite-strain model for anisotropic viscoplastic media: I - Theory. Eur. J. Mech. A /Solids 28:387-401.

Danas, K., Castaneda, P. Ponte. (2009b) A finite-strain model for anisotropic viscoplastic media: II - Applications. Eur. J. Mech. A /Solids 28:402-416.

Garrison Jr, W.M., Moody, N.R. (1987) Ductile fracture. J. Phys. Chem. Solids 48(11):1035-1074.

Geltmacher, A.B., Koss, D.A., Matic, P., Stout, M.G. (1996) A modeling study of the effect of stress state on void linking during ductile fracture. Acta Materialia 44:2201-2210.

Gologanu, M., Leblond, J., Perrin, G., Devaux, J. (1997) Recent extensions of Gurson’s model for porous ductile metals. In: Continuum Micromechanics, Springer-Verlag, Berlin, p. 61-106.

Gurson, A.L. (1977). Continuum theory of ductile rupture by void nucleation and growth - I. Yield criteria and flow rules for porous ductile media. J. Engng. Materials Technol. 99: 2-15. Hutchinson, J.W. (1973) Finite strain analysis of elastic-plastic solids and structures. In: Hartung, R.F. (Ed.), Numerical Solution of Nonlinear Structural Problems. ASME, New York, p. 17.

McClintock, F.A. (1968). A criterion for ductile fracture by growth of holes. J. Appl. Mech., 35:363-371.

Nielsen, K.L., Dahl, J. and Tvergaard, V. (2012) Collapse and coalescence of spherical voids subject to intense shearing: Studied in full 3D. Int. J. Fracture 177:97-108.

Ohno, N., Hutchinson, J.W. (1984) Plastic flow localization due to non-uniform void distribution. J. Mech. Phys. Solids 32(1):63-85. 
Pardoen, T., Hutchinson, J.W. (2000) An extended model for void growth and coalescence. J. Mech. Phys. Solids 48:2467-2512.

Pedersen, T.Ø. (1998) Remeshing in analysis of large plastic deformations. Computers and Structures 67:279-288.

Rice, J.R. and Tracey, D.M. (1969). On the ductile enlargement of voids in triaxial stress fields. J. Mech. Phys. Solids 17:201-217.

Scheyvaerts, F., Onck, P.R., Bréchet, Y., Pardoen, T. (2006) Void growth and coalescence under general loading conditions. Report, Université catholique de Louvain.

Scheyvaerts, F., Onck, P.R., Tekoglu, C., Pardoen, T. (2011) The growth and coalescence of ellipsoidal voids in plane strain under combined shear and tension. J. Mech. Phys. Solids 59:373-397.

Thomson, C.I.A., Worswick, M.J., Pilkey, A.K., Lloyd, D.J., Burger, D. (1999) Modeling void nucleation and growth within periodic clusters of particles. J. Mech. Phys. Solids 47:126.

Thomson, C.I.A., Worswick, M.J., Pilkey, A.K., Lloyd (2003) Void coalescence within periodic clusters of particles. J. Mech. Phys. Solids 51:127-146.

Tinet, H., Klöcker, H., Le Coze, J. (2004) Damage analysis during hot deformation of a resulfurized stainless steel. Acta Materialia 52:3825-3842.

Tvergaard, V. (1976) Effect of thickness inhomogeneities in internally pressurized elasticplastic spherical shells. J. Mech. Phys. Solids 24:291.

Tvergaard, V. (1981) Influence of voids on shear band instabilities under plane strain conditions. Int. J. Fracture, 17:389-407.

Tvergaard, V. (1990) Material failure by void growth to coalescence. Advances in Applied Mechanics 27:83-151.

Tvergaard, V. (1997) Studies of void growth in a thin ductile layer between ceramics, Computational Mechanics 20:186-191.

Tvergaard, V. (2004) On fatigue crack growth in ductile materials by cracktip blunting. J. Mech. Phys. Solids 52:2149-2166.

Tvergaard, V. (2007) Discrete modelling of ductile crack growth by void growth to coalescence. Int. J. Fracture 148:1-12.

Tvergaard, V. (2008) Shear deformation of voids with contact modeled by internal pressure. Int. J. Mech. Sci. 50:1459-1465.

Tvergaard, V. (2009) Behaviour of voids in a shear field. Int. J. Fracture 158:41-49. 
Tvergaard, V. (2012) Effect of stress-state and spacing on voids in a shear-field. Int. J. Solids Structures 49:3047-3054.

Tvergaard, V., Hutchinson, J.W. (2002) Two mechanisms of ductile fracture: void by void growth versus multiple void interaction. Int. J. Solids Struct. 39:3581-3597.

Tvergaard, V., Needleman, A. (1984) Analysis of the cup-cone fracture in a round tensile bar. Acta Metall., 32:157-169.

Tvergaard, V., Needleman, A. (1992) Effect of crack meandering on dynamic, ductile fracture. J. Mech. Phys. Solids, 40:447-471.

Tvergaard, V., Needleman, A. (2006) Three dimensional microstructural effects on plane strain ductile crack growth. Int. J. Solids Structures, 43:6165-6179. 


\section{Figure captions:}

Fig. 1. Coordinates and dimensions, for a case with $N_{v}=5$. (a) Region near voids. (b) The cell analysed.

Fig. 2. Example of an initial mesh near voids, for a case with $N_{v}=5$.

Fig. 3. Curves of normalized stress or void volume vs. logarithmic strain, for the stress ratio $\kappa=0.25$ and for different values $N_{v}$ of the number of voids in the cluster. (a) True tensile stress $\Sigma_{22}$ normalized by the initial yield stress. (b) Total void volume $V$ normalized by the initial volume.

Fig. 4. Deformed mesh for the computation with $\kappa=0.25$ and $N_{v}=5$, at $\varepsilon_{2}=0.174$ and $V / V_{0}=26.8$.

Fig. 5. Deformed mesh for the computation with $\kappa=0.25$ and $N_{v}=5$, at $\varepsilon_{2}=0.227$ and $V / V_{0}=1426$.

Fig. 6. Curves of normalized stress or void volume vs. logarithmic strain, for different values of the stress ratio $\kappa$ and for different values of $N_{v}$. (a) True tensile stress $\Sigma_{22}$ normalized by the initial yield stress. (b) Total void volume $V$ normalized by the initial volume.

Fig. 7. Curves of normalized stress or void volume vs. logarithmic strain, for $\kappa=0.25$. Different values of the spacing between voids and different values of $N_{v}$ are considered. (a) True tensile stress $\Sigma_{22}$ normalized by the initial yield stress. (b) Total void volume $V$ normalized by the initial volume.

Fig. 8. Curves of normalized stress or void volume vs. logarithmic strain, for $\kappa=0.25$. Different values of the strain hardening exponent $n$ and different values of $N_{v}$ are considered . (a) True tensile stress $\Sigma_{22}$ normalized by the initial yield stress . (b) Total void volume $V$ normalized by the initial volume. 


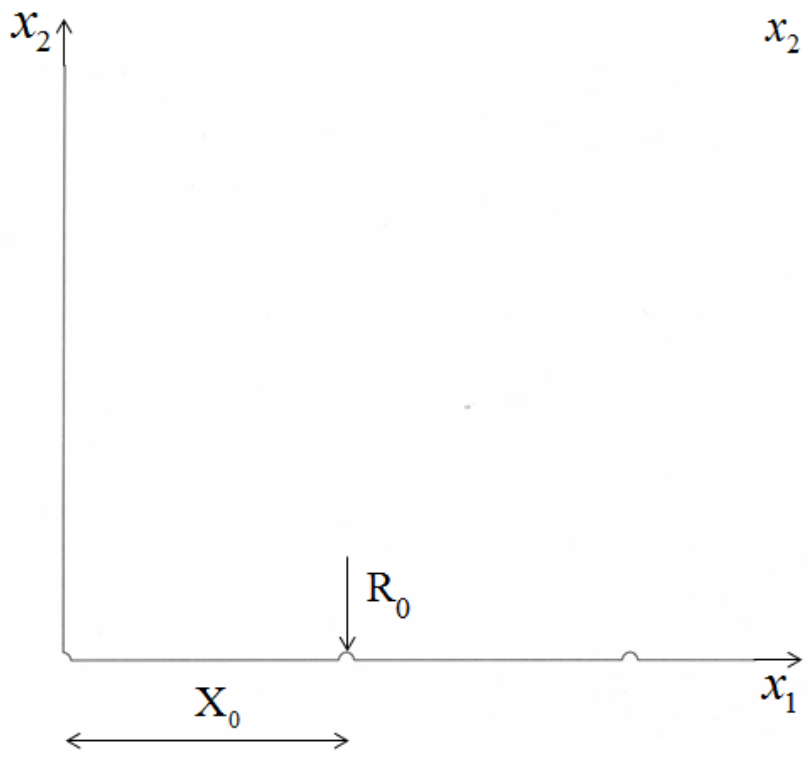

(a)

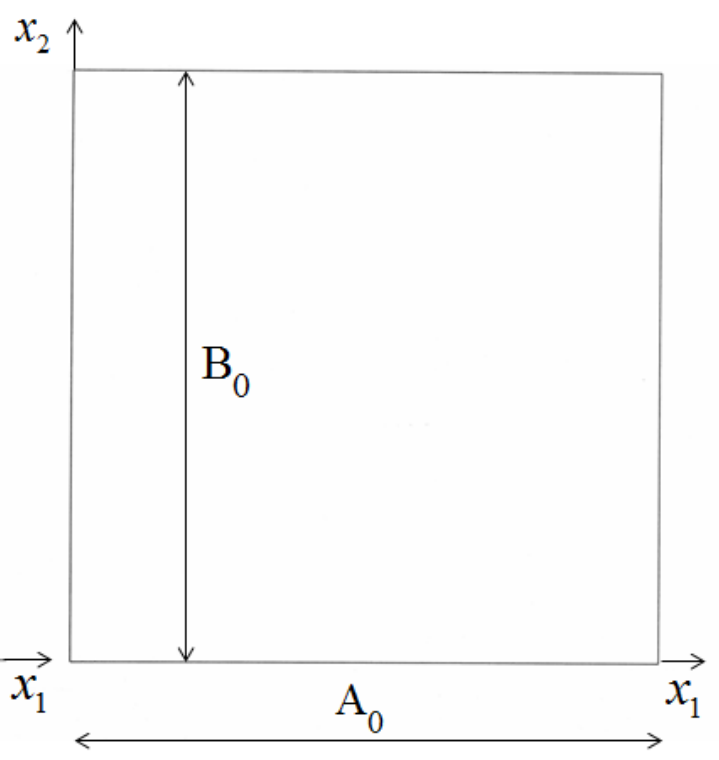

(b)

Fig. 1. Coordinates and dimensions, for a case with $N_{v}=5$. (a) Region near voids. (b) The cell analysed.

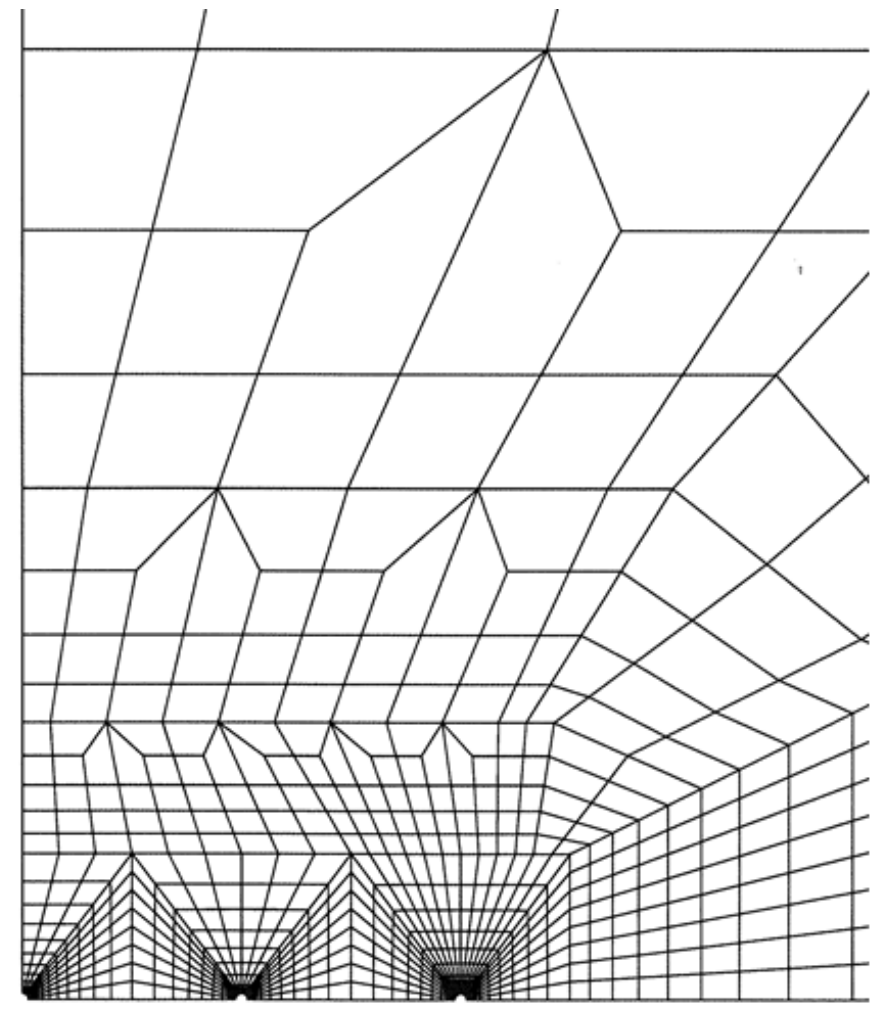

Fig. 2. Example of an initial mesh near voids, for a case with $N_{v}=5$. 

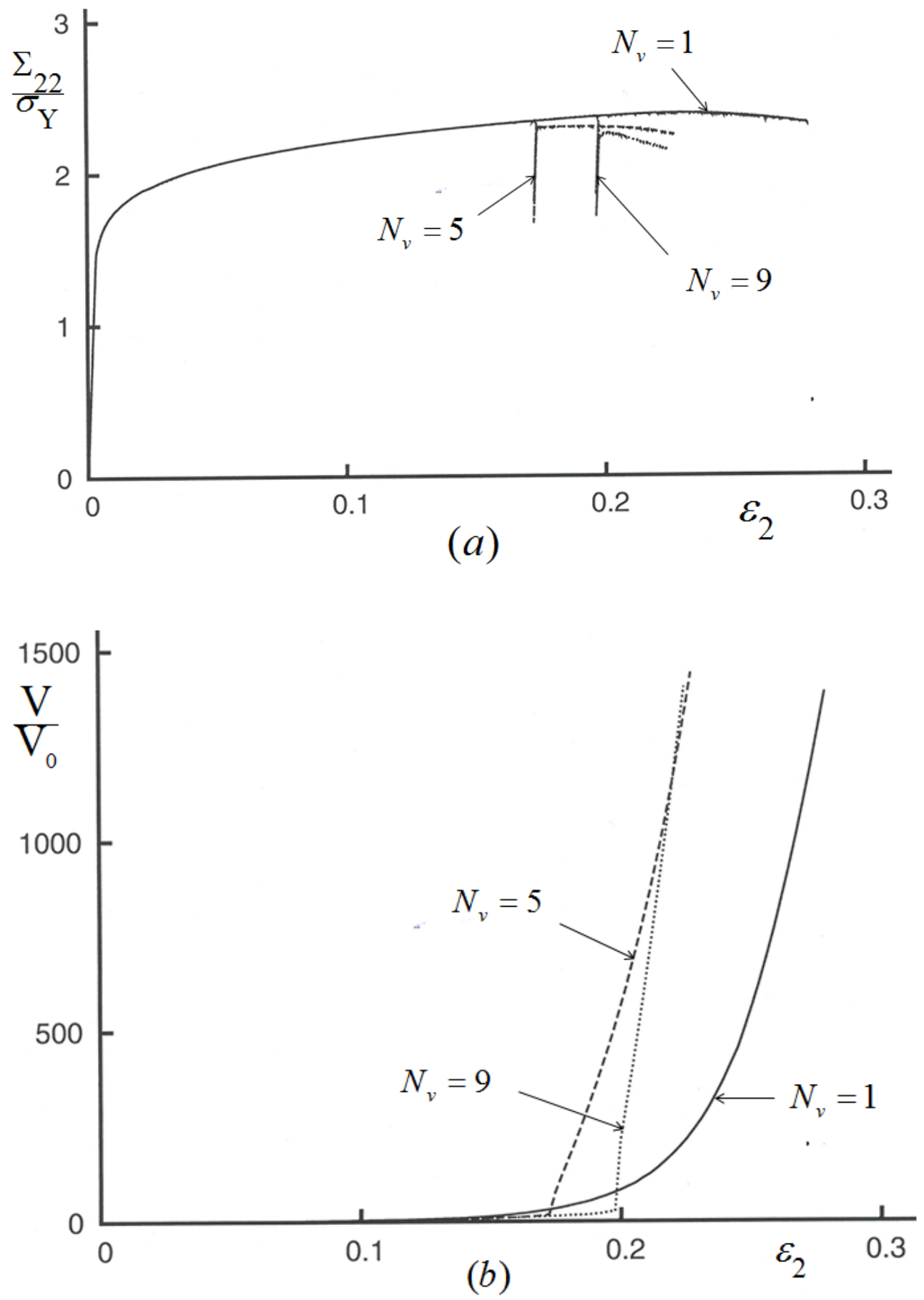

Fig. 3. Curves of normalized stress or void volume vs. logarithmic strain, for the stress ratio $\kappa=0.25$ and for different values $N_{v}$ of the number of voids in the cluster. (a) True tensile stress $\Sigma_{22}$ normalized by the initial yield stress. (b) Total void volume $V$ normalized by the initial volume. 


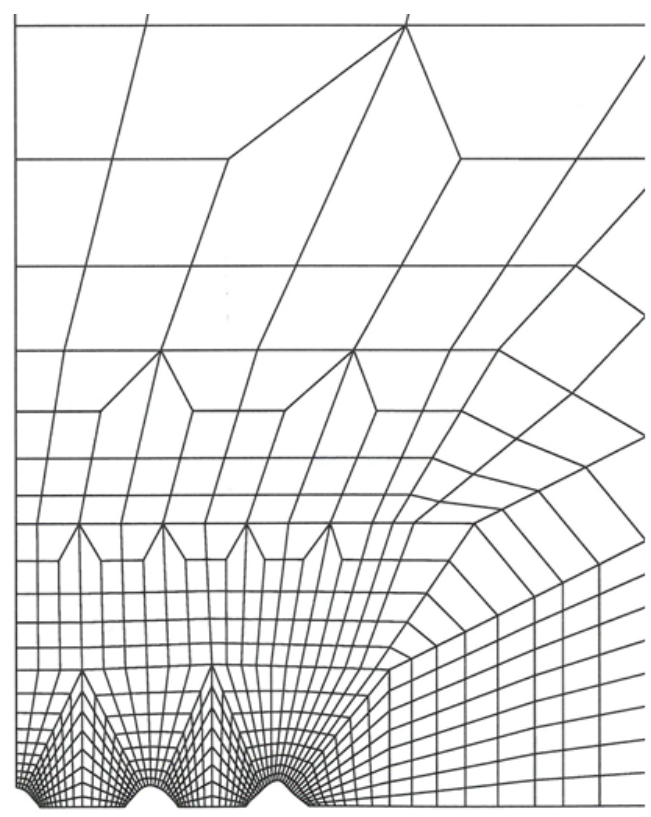

Fig. 4. Deformed mesh for the computation with $\kappa=0.25$ and $N_{v}=5$, at $\varepsilon_{2}=0.174$ and $V / V_{0}=26.8$.

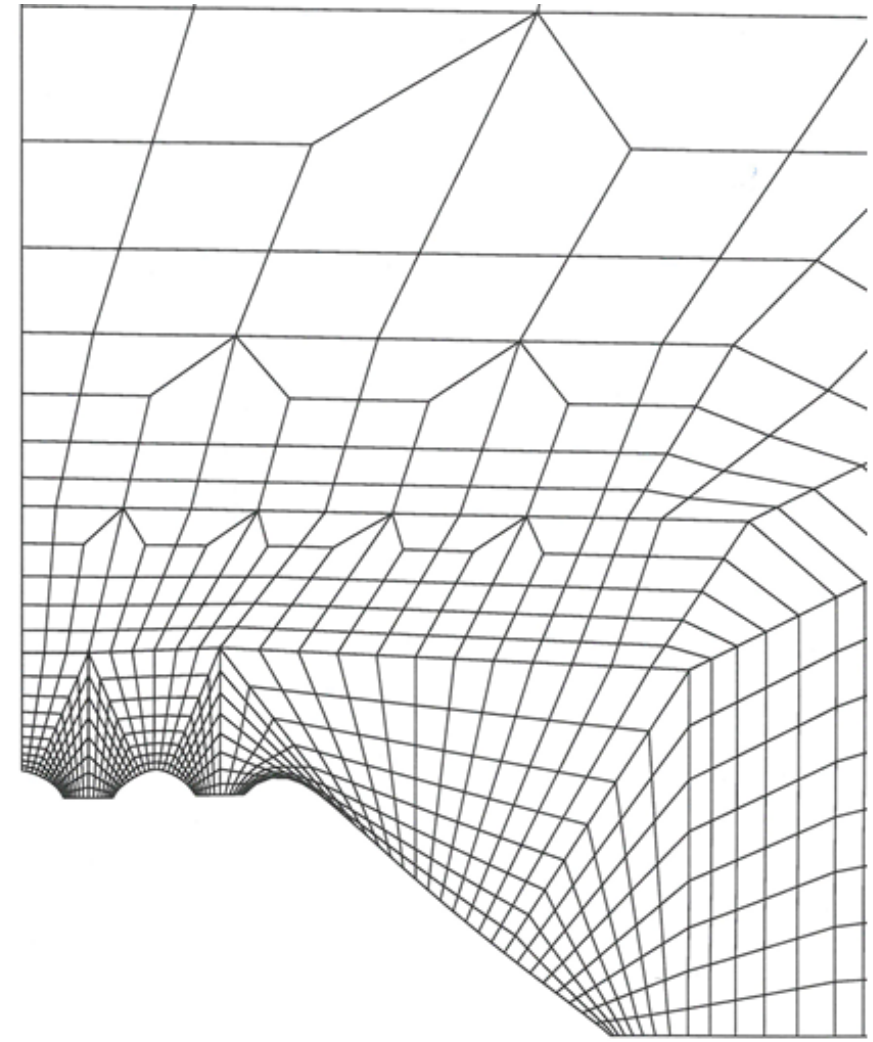

Fig. 5. Deformed mesh for the computation with $\kappa=0.25$ and $N_{v}=5$, at $\varepsilon_{2}=0.227$ and $V / V_{0}=1426$. 


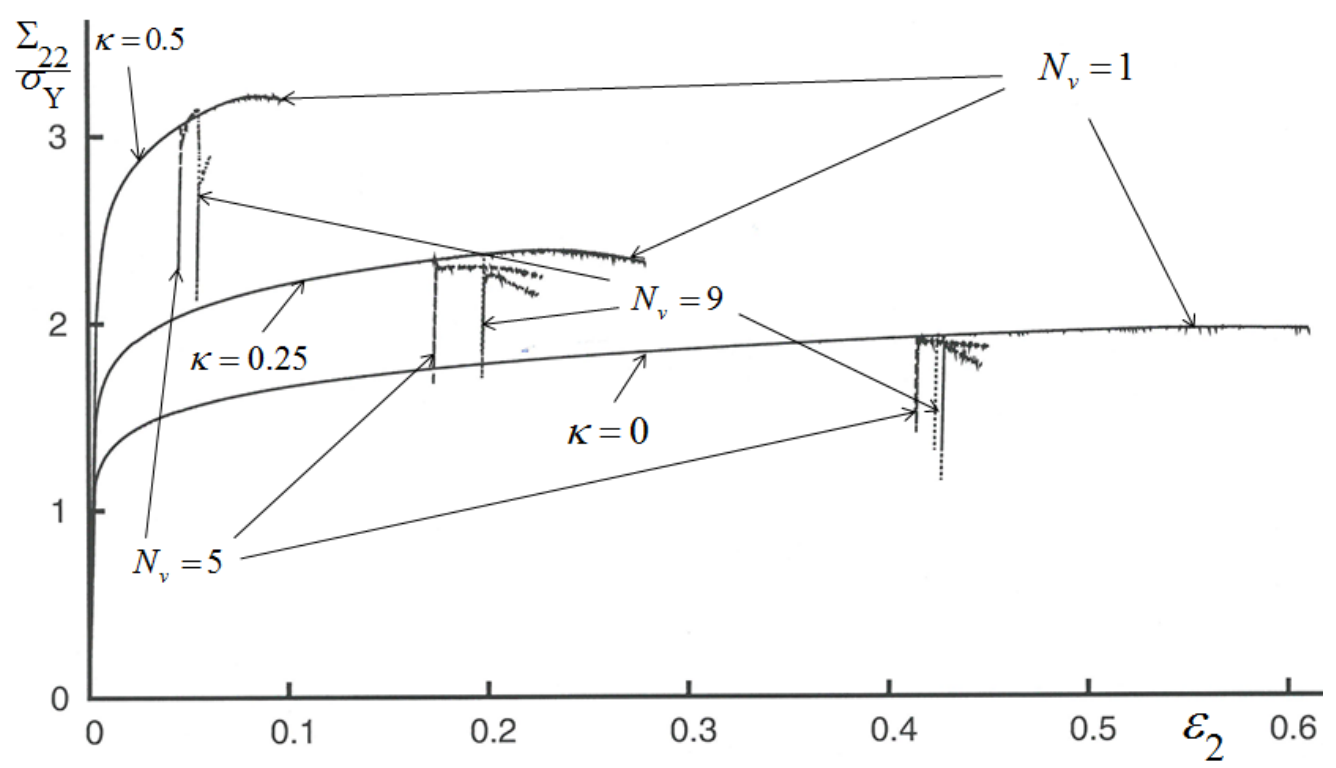

(a)

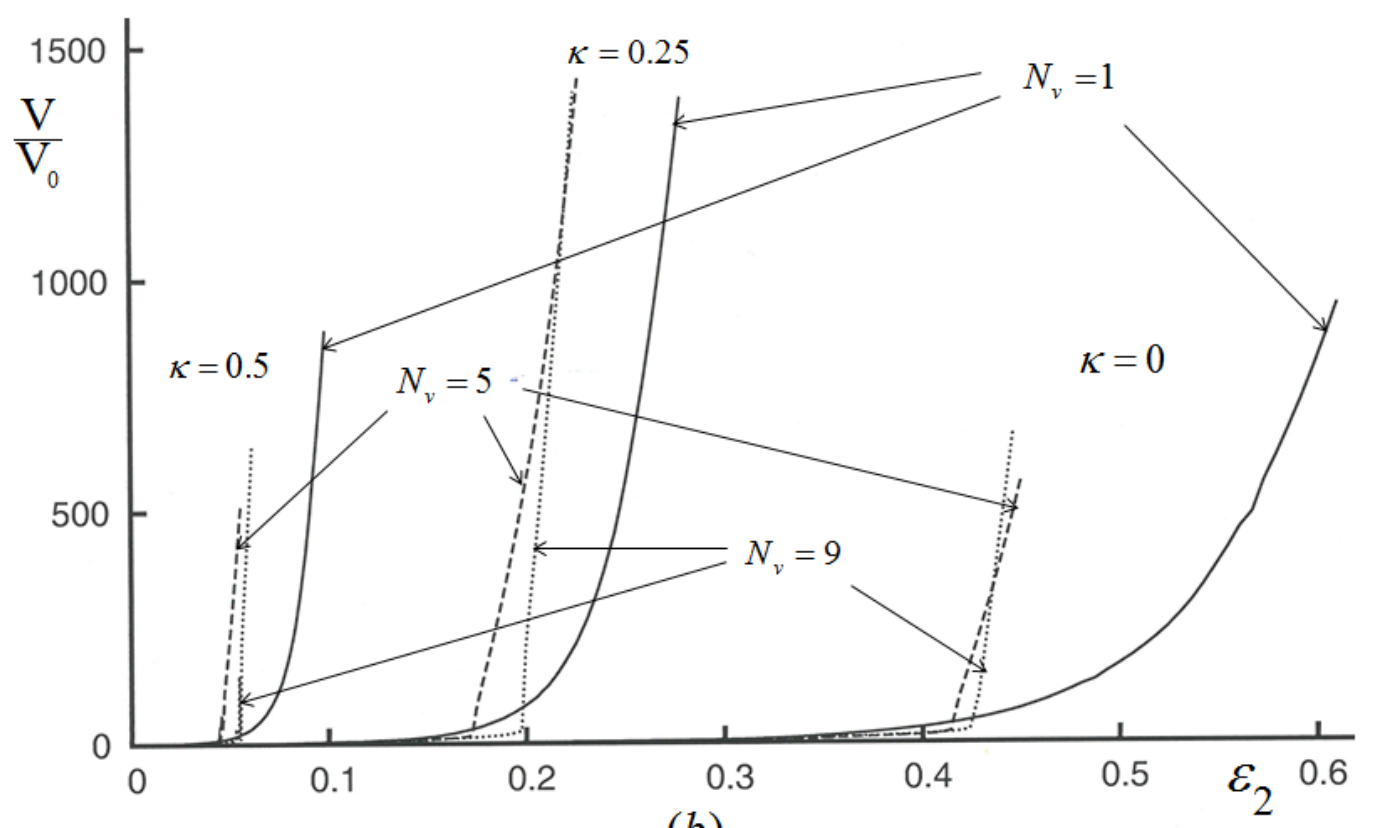

(b)

Fig. 6. Curves of normalized stress or void volume vs. logarithmic strain, for different values of the stress ratio $\kappa$ and for different values of $N_{v}$. (a) True tensile stress $\Sigma_{22}$ normalized by the initial yield stress. (b) Total void volume $V$ normalized by the initial volume. 

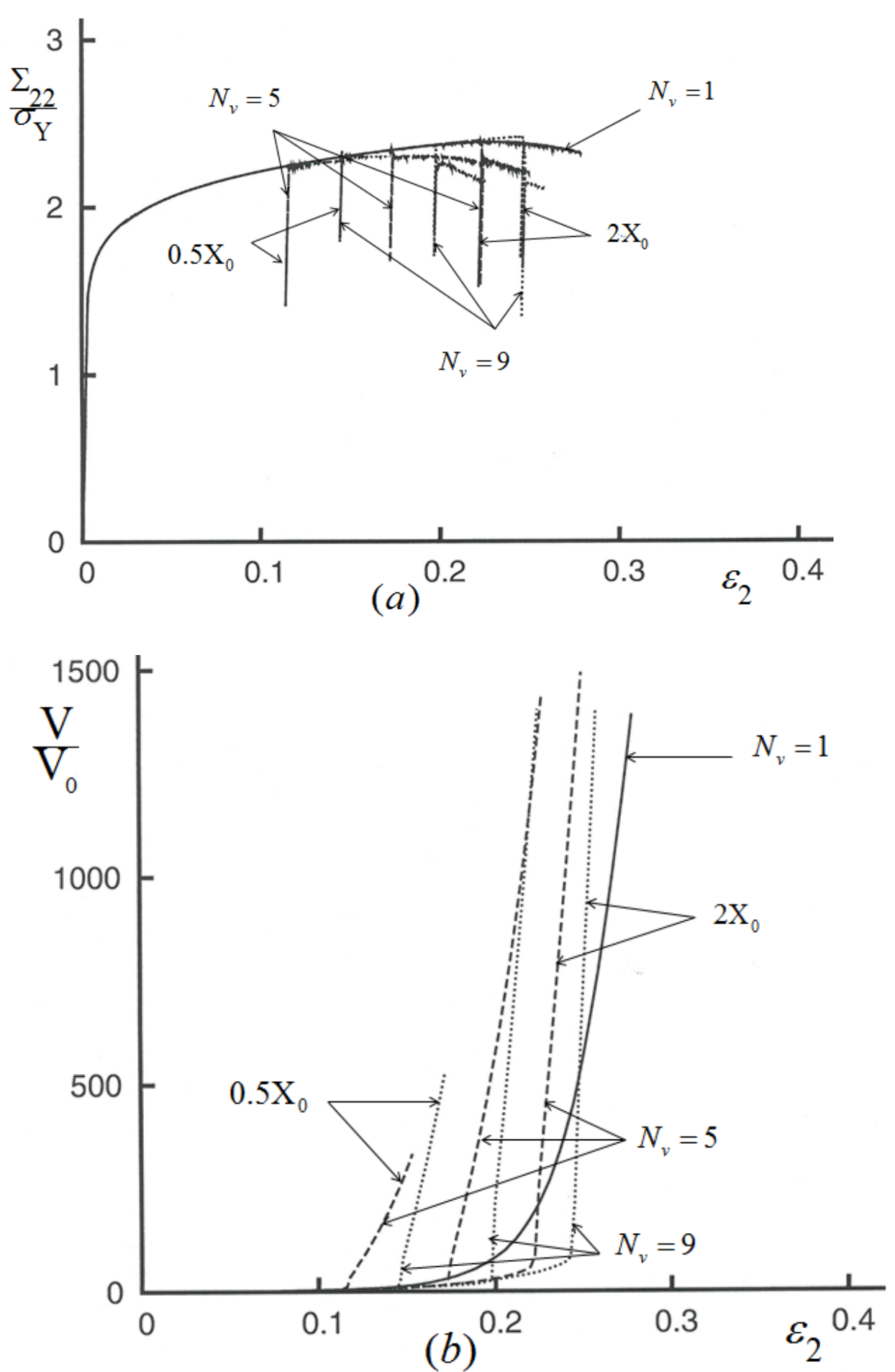

Fig. 7. Curves of normalized stress or void volume vs. logarithmic strain, for $\kappa=0.25$. Different values of the spacing between voids and different values of $N_{v}$ are considered. (a) True tensile stress $\Sigma_{22}$ normalized by the initial yield stress. (b) Total void volume $V$ normalized by the initial volume. 

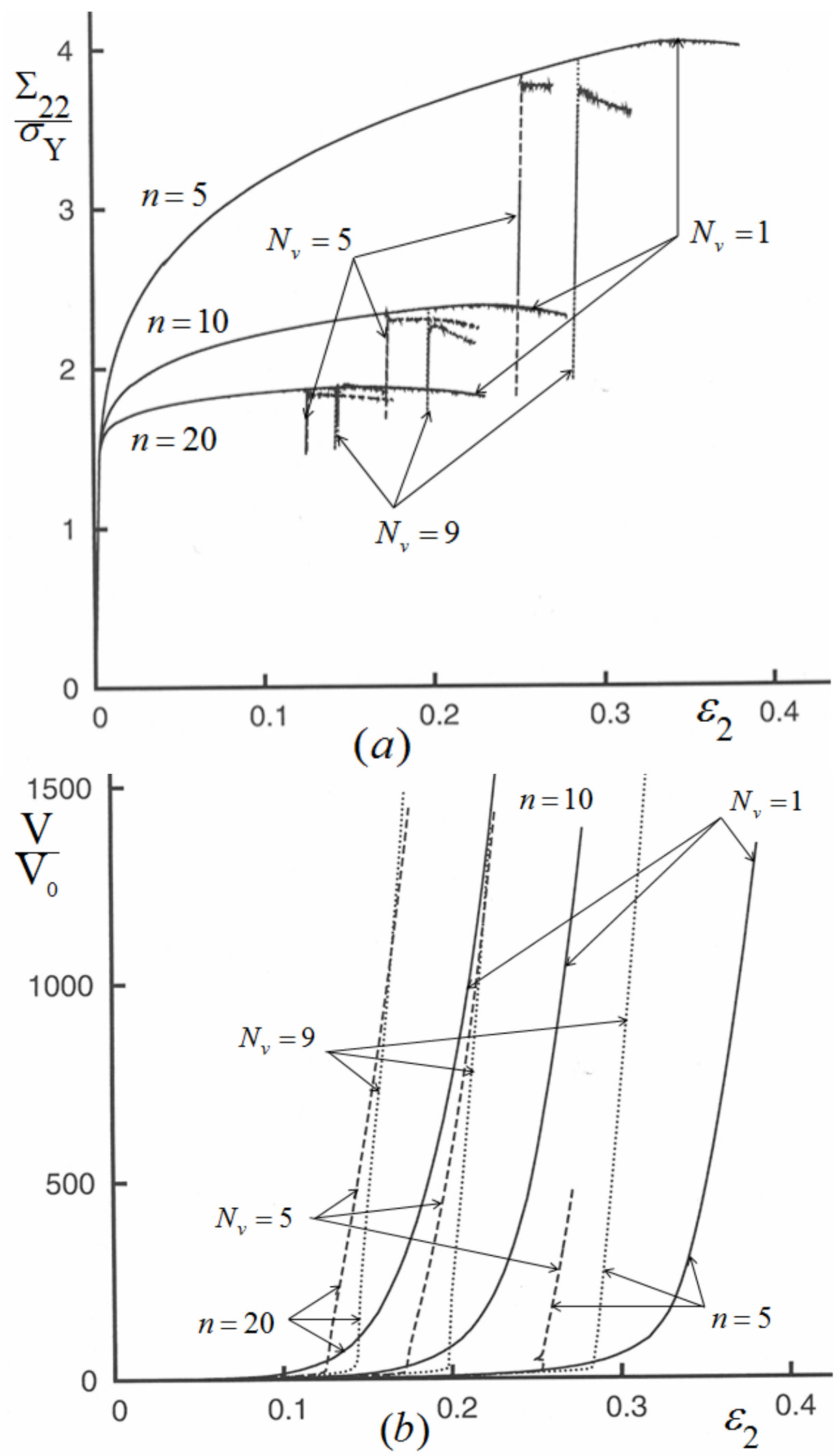

Fig. 8. Curves of normalized stress or void volume vs. logarithmic strain, for $\kappa=0.25$. Different values of the strain hardening exponent $n$ and different values of $N_{v}$ are considered . (a) True tensile stress $\Sigma_{22}$ normalized by the initial yield stress . (b) Total void volume $V$ normalized by the initial volume. 